\title{
Renal Afferents
}

\author{
Alissa A. Frame ${ }^{1}$ - Casey Y. Carmichael ${ }^{1} \cdot$ Richard D. Wainford $^{1}$
}

Published online: 5 September 2016

(C) The Author(s) 2016. This article is published with open access at Springerlink.com

\begin{abstract}
Purpose of Review The etiology of hypertension, a critical public health issue affecting one in three US adults, involves the integration of the actions of multiple organ systems, including the renal sympathetic nerves. The renal sympathetic nerves, which are comprised of both afferent (sensory input) and efferent (sympathetic outflow) arms, have emerged as a major potential therapeutic target to treat hypertension and disease states exhibiting excess renal sympathetic activity. Recent Findings This review highlights recent advances in both clinical and basic science that have provided new insight into the distribution, function, and reinnervation of the renal sympathetic nerves, with a focus on the renal afferent nerves, in hypertension and hypertension-evoked disease states including salt-sensitive hypertension, obesity-induced hypertension, and chronic kidney disease.

Summary Increased understanding of the differential role of the renal afferent versus efferent nerves in the pathophysiology of hypertension has the potential to identify novel targets and refine therapeutic interventions designed to treat hypertension.
\end{abstract}

This article is part of the Topical Collection on Hypertension and the Brain

Richard D. Wainford

rwainf@bu.edu

Alissa A. Frame

aframe@bu.edu

Casey Y. Carmichael

cascar@bu.edu

1 Department of Pharmacology \& Experimental Therapeutics and The Whitaker Cardiovascular Institute Boston University School of Medicine, 72 East Concord St, Boston 02118, MA, USA
Keywords Renal afferent nerves $\cdot$ Renal efferent nerves · Hypertension $\cdot$ Radiofrequency ablation $\cdot$ Renal denervation . Sympathetic nervous system

\section{Introduction}

Hypertension, which affects one third of the US adult population and approximately $20 \%$ of adults worldwide, is a major risk factor for adverse cardiovascular events - significantly contributing to the epidemic of cardiovascular and kidney disease, stroke, premature death, and disability [1]. Hypertension is a complex multifactorial disease involving the integration of multiple regulatory systems and our understanding of the pathogenesis of this disease state remains incomplete. Recent studies have explored the renal sympathetic nerve-mediated mechanisms, both afferent and efferent nerve based, in the long-term regulation of blood pressure as increasing basic science and clinical evidence implicates the renal nerves as a contributor to the pathophysiology of hypertension [2-4]. Removal or interruption of the afferent and efferent renal sympathetic nerves, which are located together in the adventitia of the renal arteries, has been shown to reduce blood pressure through multiple mechanisms. Reduction in efferent renal nerve traffic to the kidney prevents sympathetic nervous system-mediated (1) increased activity of the reninangiotensin-aldosterone system, (2) renal sodium reabsorption, and (3) reduced renal blood flow [5]. In contrast, removal of afferent renal nerve traffic impacts the reno-renal reflexes to modulate central sympathetic outflow to peripheral organs (e.g., kidneys, heart) and the vasculature. The sensory renal afferent nerves, located predominantly in the renal pelvis [5], are chemo- and mechanosensitive and upon activation can elicit either (1) an inhibitory reno-renal reflex to evoke renal sympathoinhibition $[5,6]$ or (2) an excitatory reno-renal reflex 
to evoke sympathoexcitation [6-8]. Highlighting the potential importance of the renal sympathetic nerves in regulating blood pressure, it has been suggested that more than $50 \%$ of clinical hypertension cases can be categorized as neurogenic essential hypertension and feature impaired regulation of the sympathetic nervous system [9]. Therefore, a more complete understanding of the role(s) of the afferent renal nerves, which have a profound impact on central sympathetic outflow, in both health and disease will potentially reveal novel targets and treatment paradigms for hypertension. The purpose of this review is to highlight recent advancements in our understanding of the actions of the afferent renal nerves in the etiology and pathogenesis of hypertension.

\section{Catheter-Based Radiofrequency Renal Denervation}

The use of catheter-based radiofrequency renal denervation (RFRD), which interrupts both the afferent and efferent renal nerves, as an interventional approach to treat hypertension has been under investigation in multiple clinical trials for the past several years. The SYMPLICITY HTN-1 and HTN-2 trials reported the blood pressure-lowering effects of RFRD are sustained for at least a 36-month period in relatively small populations with severe treatment resistant hypertension [4, 10]. In contrast, the SYMPLICITY HTN-3 trial failed to show a significant reduction in systolic blood pressure between sham and RFRD groups 6 months post treatment [11], highlighting the pressing need to deepen our understanding of the roles of the afferent and efferent nerves in blood pressure regulation. The SYMPLICITY HTN-3 trial has several advantages over SYMPLICITY HTN-1 and HTN-2, including a larger sample size, inclusion of a control sham procedure, and a single-blinded experimental design. This raises the possibility, which remains to be fully investigated, that the long-term antihypertensive effects observed in the highly controlled small-scale HTN-1 and HTN-2 trials may not be reproducible in the general population. The issues relating to the design and conductance of SYMPLICITY HTN-3 are beyond the scope of this review-however, the effectiveness of the RFRD procedures conducted in this study have significant implications for the reported negative outcome. In this study, on average, operators performed approximately 3 RFRD procedures without previous experience. This raises the significant issue that lack of operator experience may have impacted the results through incomplete denervation [12], a profound issue given the lack of a standardized accepted test for the effective destruction of the renal sympathetic nerves during the procedure [13]. Furthermore, the DENERHTN trial, in which RFRD plus a standardized stepped-care antihypertensive treatment (SSAHT) decreased blood pressure more than identical SSAHT alone, highlights the potential importance and clinical applicability of RFRD as a therapeutic approach
[14・•]. Ultimately, the interpretation of conflicting data from clinical RFRD trials is complicated by the current absence of a clinical test confirming the effective destruction of the renal sympathetic nerves [13], and the degree of denervation required for therapeutic benefit in humans has not been investigated.

Subsequent post hoc analysis of the SYMPLICITY HTN-3 trial has revealed important predictors of the blood pressure response to RFRD which are likely to guide future investigations pursuing RFRD. Supporting the impact of patient medication, as revealed in DENERHTN, aldosterone antagonist use and non-use of vasodilators was a positive predictor of RFRD success. Highlighting the pivotal role of how RFRD is performed, these analyses revealed that a higher number of radiofrequency ablations and RFRD delivered in a fourquadrant pattern resulted in greater blood pressure reduction [15•]. Two recent basic science studies have suggested that modifications to the RFRD technique may improve efficacy of the procedure. An elegant study performed in swine conducted RFRD at three sites, near the ostium, in the main renal artery near the bifurcation, and in the renal artery extrarenal branches [16]. This study revealed the greatest reduction in renal NE content was observed following RFRD in the extrarenal arterial branches, a result that may reflect that this location had the greatest density of renal nerves and the greatest number of nerves in proximity to the lumen. A second study conducted in dogs suggests that electrical stimulation of the renal nerves, which evoked rapid changes in blood pressure via central mechanisms, could represent a response that could (1) identify the sites at which RFRD should be applied and (2) be applied post RFRD to confirm efficacy of RFRD $[17 \bullet \bullet]$. The validation of such a test is critical given the conflicting results of clinical RFRD trials and the observation that a threshold of $90 \%$ surgical renal denervation is required for therapeutic benefit in animal models. At present it remains unknown if more extensive RFRD of both the afferent and efferent renal nerves and conductance of RFRD closer to the kidney would result in more profound and consistent reductions in blood pressure. However, increased ablation sites or increased energy frequency may evoke adverse effects not observed under the current treatment protocol.

\section{Renal Afferent and Efferent Nerve Distribution and Reinnervation}

The unmet need to better understand the physiology and anatomy of the renal sympathetic nerves and characterize the impact of reinnervation post renal sympathetic nerve denervation has led to several recent advances. A pioneering study conducted in human autopsy subjects has shed new insight into the anatomical distribution of the peri-arterial sympathetic renal nerves located around the renal arteries [18]. This 
important study has revealed the anatomical distribution of the renal nerves with a clear predominance of efferent versus afferent nerve fibers and a decreasing presence of nerve fibers from proximal to distal locations. Further, it was revealed that within the distal regions (closest to the kidney), approximately $75 \%$ of nerves are within $3 \mathrm{~mm}$ of the lumen, well within the range of RFRD, which generates lesions of 4-mm diameter. This suggests, as confirmed in a recent study conducted in swine [16], that greater RFRD efficacy is likely when ablation is conducted distally. Significantly, there was a difference observed in renal nerve anatomy between normotensive versus hypertensive subjects [18]. Two recent postmortem studies support the hypothesis that in renal arteries with a large diameter, renal nerve innervation is located further from the lumen, which would reduce efficacy of RFRD and require greater energy to produce significant ablation $[19,20]$. The most recent study [20] again supports the growing body of evidence that the nerves are closer to the lumen in distal versus proximal regions. This increased knowledge of renal nerve anatomy will facilitate the refinement of current RFRD techniques, with future directions including RFRD at more distal locations and the adoption of a circumferential versus interrupted pattern of ablation.

As it is not yet possible to assess reinnervation in human subjects, a recent study conducted in normal Sprague-Dawley rats utilized immunohistochemistry to investigate the time course of renal reinnervation following surgical renal denervation $(\mathrm{RDN})$ [21]. In this study, peptide markers of both afferent (substance $\mathrm{P}$, calcitonin gene-related peptide) and efferent renal nerves (tyrosine hydroxylase, neuropeptide $\mathrm{Y}$ ) were restored 9-12 weeks post denervation. While these results should be confirmed using neuroanatomic markers to verify the renal nerves as the source of the peptide markers, this study raises the intriguing, yet to be addressed, question of the functionality of the renal nerves following reinnervation in this setting. A recent study in sheep partially addresses this question by reporting that physiological responses to electrical stimulation of the renal nerves are abolished immediately following RFRD in normotensive sheep but are restored at 5.5 and 11 months post RFRD. Importantly, these functional changes are mirrored by the initial loss and subsequent restoration of peptide markers of both afferent and efferent renal nerves [22•]. Given the prolonged time frame of blood pressure reduction post RFRD in human patients [4, 10], it is highly likely that reinnervation has occurred without altering the blood pressure phenotype. A significant and clinically relevant question that remains unanswered is the functional status of reinnervated nerves. Are the responses of the regrown renal afferent nerves restored to normal in hypertensive animals or human subjects? Do regrown efferent nerves exhibit normal regulation of sodium, renin, and renal hemodynamics? Is there resetting of central nervous system mechanisms to alter sympathetic outflow following RFRD?

\section{Selective Renal Afferent Nerve Ablation}

As previously discussed, surgical denervation and RFRD removes the influence of both the afferent and efferent renal sympathetic nerves - preventing the dissection of the individual actions of each arm. To facilitate the dissection of the role of the renal afferent nerves, a novel method of selective ablation of the renal afferent nerves by periaxonal application of capsaicin has been developed in rats [23••]. This technique selectively disrupts the renal afferent but not efferent renal nerves (confirmed pharmacologically and immunohistochemically), and supports recent studies on the timeline of nerve regrowth, with afferent nerve reinnervation suggested by the return of calcitonin gene-related peptide to $50 \%$ of control levels within 7 weeks [23••]. However, the time course of renal afferent nerve reinnervation requires further investigation using neuroanatomical markers. These studies provide a new opportunity to examine the contribution of the afferent renal nerves to multiple pathophysiological states. Studies conducted using this technique suggest that the renal afferent nerves are not essential to maintain normotension and sodium balance in response to graded chronic alterations in dietary sodium intake Sprague-Dawley rats. In contrast, the renal afferent nerves play a role in the full development of deoxy-corticosterone acetate-salt hypertension, with afferent nerve ablation attenuating the observed hypertension by approximately $50 \%[23 \bullet \bullet$. Extending investigations into the role(s) of the renal sympathetic nerves in the development of salt-sensitive hypertension, bilateral RDN conducted after the establishment of hypertension reduces blood pressure in the Dahl salt-sensitive (DSS) rat model [24]. A follow-up study confirmed that bilateral RDN reduces blood pressure in established short- and long-term DSS hypertension, and that this response reflects an efferent renal nerve effect as renal afferent nerve removal did not reduce blood pressure in hypertensive DSS rats [25]. These new studies, extending findings from the 1980s in which bilateral RDN prior to high salt intake did not attenuate the development of DSS hypertension, suggest that the underlying cause of hypertension, and the role of the renal nerves, may be different during developing versus established hypertension.

Bilateral RDN, but not selective renal afferent nerve ablation, attenuates angiotensin-II hypertension and inflammation, further suggesting that the differential impact of the afferent versus efferent nerves on blood pressure regulation may depend on the underlying cause of hypertension [26]. The differential responses observed to RDN and selective renal afferent nerve ablation in these recent studies highlight the unique mechanistic information that can be derived using the novel renal afferent ablation technique, and future studies are required to assess the roles of the renal afferent nerves in other hypertension paradigms (e.g., obesity-induced hypertension, 
neurogenic hypertension) and settings involving excess sympathetic nerve activity (e.g., heart failure).

\section{The Renal Afferent Nerves and Heart Failure}

In the pathophysiology of heart failure, which can arise as a result of hypertension-evoked heart disease, reduced cardiac output and organ perfusion evokes multiple compensatory mechanisms including activation of the sympathetic nervous system. Increased efferent renal nerve activity, which evokes renal sodium and water retention, renin release, and renal vasoconstriction, is widely accepted to contribute to renal dysfunction in heart failure [27]. Further, recent studies in conscious sheep have identified the hypothalamic paraventricular nucleus (PVN) as a central site that regulates increased renal sympathetic outflow in heart failure [28]. In contrast, the role(s) of the renal afferent nerves in the pathophysiology of heart failure remains largely unexplored. Recent studies from the Patel laboratory reported that there is increased activity of RVLM-projecting PVN neurons in a rat model of chronic heart failure [29]. Advancing this work, the same authors have revealed that there is a neural connection from the PVN to the RVLM that is activated by direct stimulation of the renal afferent nerves [30॰]. These data illustrate that afferent output from the kidney is integrated with PVN preautonomic neurons, significantly enhancing our mechanistic understanding of the actions of the renal afferent nerves. This raises the untested hypothesis that this novel renal afferent nerve activated neural circuit may play a critical role in the modulation of sympathetic outflow in heart failure. It has been previously established that the inhibitory mechanoreceptor reno-renal reflex is attenuated in heart failure [5]. However, the modulation of the excitatory chemo-reflex in heart failure remains to be established.

The impact of RDN on heart failure has been investigated in multiple experimental models and in clinical studies in the last several years. Post-myocardial infarction bilateral RDN improves cardiac function in rats [31]. In rat models of heart failure, bilateral RDN has been demonstrated to (1) attenuate renal sodium reabsorption via a $\mathrm{Na}-\mathrm{K}-2 \mathrm{Cl}$ dependent pathway when RDN is performed prior to the establishment of heart failure [32] and (2) improve cardiac function when RDN is conducted during established heart failure [33]. In larger animal models of pacing-induced heart failure, bilateral RDN prior to heart failure induction improved cardiac function [34]. Suggesting the potential importance of bilateral RDN, unilateral RDN did not improve cardiac function despite improving cardiac autonomic balance via reductions in sympathetic outflow in a rabbit pacing-induced heart failure model [35]. In clinical trials in heart failure patients, RDN has been demonstrated to be safe and efficacious and to increase walking distance [36], reduce left ventricular mass, and increase ejection fraction [37, 38]. Collectively, these basic science and clinical data suggest that RDN represents a therapeutic approach that would benefit heart failure patients.

Ventricular arrhythmias (VAs) are the major cause of sudden cardiac death in most forms of heart disease, including hypertension-evoked heart disease [39]. It is well established that increased sympathetic activity contributes to the development and maintenance of VAs [40]. As such, recent studies have investigated the utility of RFRD, which reduces sympathetic activity, as a therapeutic approach to treat VAs. A pivotal role of the renal nerves in triggering VAs has been demonstrated by evidence that electrical activation of the renal sympathetic nerves promotes acute ischemia-induced VAs through actions on the left stellate ganglion in a canine model [41]. Further evidence generated in canine models supports a role of the renal nerves in VAs, as RDN reduces VAs during acute myocardial ischemia [42] and pacing-induced heart failure [34]. These studies suggest the mechanism underlying the attenuation of VAs following RDN is a correction of ventricular electrophysiological remodeling [34, 42]. The utility of RDN in the clinical setting as an approach to treat VAs has been explored in small populations with cardiac myopathy and treatment-resistant ventricular tachycardia. These studies have demonstrated (1) the safety of this procedure in this patient population and (2) a profound reduction in VAs [43-45]. Collectively, these data suggest that RDN represents a potential approach to treat heart failure and VAs, and that patients with identified sympathetic over activity are likely to benefit most from this approach. However, further studies to elucidate the likely differential effects of the afferent versus efferent renal nerves in these clinical paradigms would provide additional mechanistic insight.

\section{The Renal Afferent Nerves and Obesity-Induced Hypertension}

It is well established that increased sympathetic nervous system activity contributes to the development and maintenance of obesity-induced hypertension [46]. Of relevance to this review is the pivotal role of the renal sympathetic nerves in obesity-evoked hypertension. Bilateral RDN restored blood pressure to normotensive levels in dogs with established obesity-induced hypertension [47]. Significantly, confirming the validity of a catheter-based approach, RFRD lowered blood pressure in a dog model of obesity-induced hypertension despite only partial nerve ablation [48]. Based on seminal work done in the 1990s in the laboratory of Dr. Hall, it is thought that the major benefits of RDN in obesity-induced hypertension are mediated via efferent renal nerve removal versus interruption of afferent renal nerve pathways. Recent findings in obese normotensive Sprague-Dawley rats imply that neural signals originating from the kidney (presumably 
renal afferent nerve mediated) contribute to autonomic dysregulation (i.e., impaired arterial and cardiopulmonary baroreflexes) in obesity [49]. While a direct role of the afferent renal nerves in mediating signal transduction from the kidney in this paradigm requires further confirmation (e.g., selective renal afferent nerve ablation), these studies challenge the lack of a role of the renal afferent nerves in this pathophysiological condition and suggest reassessment of the impact of the renal afferent nerves in obesity-induced hypertension may yield new insight.

\section{The Renal Afferent Nerves and Chronic Kidney Disease}

Chronic kidney disease (CKD), which affects approximately $8 \%$ of the world's population over the age of 30 , can be initiated or exacerbated by hypertension. CKD exhibits the hallmarks of sympathetic activation and baroreflex dysfunction that increases with disease progression [50]. Recent studies in a rodent model of cisplatin-induced renal failure have reported inappropriate activation of renal afferent sensory nerves, which impairs the regulation of renal sympathetic outflow. The authors report that high and low pressure baroreflex regulation of renal sympathetic nerve activity and volume expansion evoked renal sympathoinhibition is attenuated in cisplatin-induced renal failure - responses that are restored following acute bilateral RDN $[6,51]$. These data suggest that in CKD, renal afferent sensory nerve activation via inflammatory pathways may evoke an excitatory reno-renal reflex to enhance renal efferent nerve activity and renal sympathoexcitation. Extending these data to a clinical setting, patients with end-stage renal disease on dialysis exhibit a marked increase in renal nerve density versus control subjects. Additionally, this study reported increased renal nerve endings present in patients with hypertensive arteriolar damage [52•]. This study did not differentiate between the presence of renal afferent versus efferent fibers but suggests RFRD may be a suitable therapeutic approach in the setting of CKD.

\section{Conclusion}

Over the last several years, our understanding of the locations and function of the renal afferent and efferent nerves has dramatically increased through novel discoveries in both basic science and clinical arenas. These findings have solidified the renal sympathetic nerves as a predominant mechanism in multiple hypertensive disease states to drive elevations in both sympathetic outflow and blood pressure. The advances outlined in this review have revealed an increasingly important role of the renal sympathetic nerves in blood pressure regulation and sympathetic outflow. These findings suggest that the site at which RFRD is performed may significantly impact the outcome in terms of blood pressure, and that the renal afferent nerves may function differently depending on the underlying causes of hypertension. The next few years will provide further delineation of the role(s) of renal afferent nerve pathways and will potentially identify cross talk of these pathways across hypertension disease states. We speculate that current and future identification of the function and anatomy of the renal afferent and efferent sympathetic nerves will generate new therapeutic targets and refinements to current catheter-based renal denervation approaches that will have broad applications in the treatment of human hypertension.

Acknowledgments This work was supported by NIH grants R01HL107330 and K02HL112718 to RDW.

\section{Compliance with Ethical Standards}

Conflict of Interest Drs. Frame, Carmichael, and Wainford declare no conflicts of interest relevant to this manuscript.

Human and Animal Rights and Informed Consent This article does not contain any studies with human or animal subjects performed by any of the authors.

Open Access This article is distributed under the terms of the Creative Commons Attribution 4.0 International License (http:// creativecommons.org/licenses/by/4.0/), which permits unrestricted use, distribution, and reproduction in any medium, provided you give appropriate credit to the original author(s) and the source, provide a link to the Creative Commons license, and indicate if changes were made.

\section{References}

Papers of particular interest, published recently, have been highlighted as:

- Of importance

•- Of major importance

1. Mozaffarian D, Benjamin EJ, Go AS, Arnett DK, Blaha MJ, Cushman M, et al. Heart Disease and Stroke Statistics-2016 Update: a report from the American Heart Association. Circulation. 2016;133(4):e38-360. doi:10.1161/CIR.0000000000000350.

2. Johns EJ. Autonomic regulation of kidney function. Handb Clin Neurol. 2013;117:203-14. doi:10.1016/B978-0-444-53491$0.00017-1$

3. Johns EJ, Abdulla MH. Renal nerves in blood pressure regulation. Curr Opin Nephrol Hypertens. 2013;22(5):504-10. doi:10.1097 /MNH.0b013e3283641a89.

4. Krum H, Schlaich MP, Sobotka PA, Bohm M, Mahfoud F, RochaSingh K, et al. Percutaneous renal denervation in patients with treatment-resistant hypertension: final 3-year report of the Symplicity HTN-1 study. Lancet. 2014;383(9917):622-9. doi:10.1016/S0140-6736(13)62192-3. 
5. Kopp UC. Role of renal sensory nerves in physiological and pathophysiological conditions. Am J Physiol Regul Integr Comp Physiol. 2015;308(2):R79-95.

6. Goulding NE, Johns EJ. Neural regulation of the kidney function in rats with cisplatin induced renal failure. Front Physiol. 2015;6:192. doi:10.3389/fphys.2015.00192.

7. Barry EF, Johns EJ. Intrarenal bradykinin elicits reno-renal reflex sympatho-excitation and renal nerve-dependent fluid retention. Acta Physiol (Oxf). 2015;213(3):731-9. doi:10.1111/apha.12420.

8. Johns EJ. The neural regulation of the kidney in hypertension and renal failure. Exp Physiol. 2014;99(2):289-94. doi:10.1113 /expphysiol.2013.072686.

9. Parati G, Esler M. The human sympathetic nervous system: its relevance in hypertension and heart failure. Eur Heart J. 2012;33(9):1058-66. doi:10.1093/eurheartj/ehs041.

10. Esler MD, Bohm M, Sievert H, Rump CL, Schmieder RE, Krum H, et al. Catheter-based renal denervation for treatment of patients with treatment-resistant hypertension: 36 month results from the SYMPLICITY HTN-2 randomized clinical trial. Eur Heart J. 2014;35(26):1752-9. doi:10.1093/eurheartj/ehu209.

11. Bhatt DL, Kandzari DE, O'Neill WW, D'Agostino R, Flack JM, Katzen BT, et al. A controlled trial of renal denervation for resistant hypertension. N Engl J Med. 2014;370(15):1393-401. doi:10.1056 /NEJMoa1402670.

12. Luscher TF, Mahfoud F. Renal nerve ablation after SYMPLICITY HTN-3: confused at the higher level? Eur Heart J. 2014;35(26): 1706-11. doi:10.1093/eurheartj/ehu195.

13. Mahfoud F, Bhatt DL. Catheter-based renal denervation: the black box procedure. JACC Cardiovasc Interv. 2013;6(10):1092-4. doi:10.1016/j.jcin.2013.09.001.

14.• Azizi M, Sapoval M, Gosse P, Monge M, Bobrie G, Delsart P, et al. Optimum and stepped care standardised antihypertensive treatment with or without renal denervation for resistant hypertension (DENERHTN): a multicentre, open-label, randomised controlled trial. Lancet. 2015;385(9981):1957-65. doi:10.1016/S0140-6736 (14)61942-5. This study validates the use or radio-frequemcy ablation as a therapeuitc approach to lower blood pressure in patients on a stepped care standardised antihypertensive treatment paradigm.

15. Kandzari DE, Bhatt DL, Brar S, Devireddy CM, Esler M, Fahy M, et al. Predictors of blood pressure response in the SYMPLICITY HTN-3 trial. Eur Heart J. 2015;36(4):219-27.

16. Henegar JR, Zhang Y, Hata C, Narciso I, Hall ME, Hall JE. Catheter-based radiofrequency renal denervation: location effects on renal norepinephrine. Am J Hypertens. 2015;28(7):909-14. doi:10.1093/ajh/hpu258.

17.• Chinushi M, Izumi D, Iijima K, Suzuki K, Furushima H, Saitoh O, et al. Blood pressure and autonomic responses to electrical stimulation of the renal arterial nerves before and after ablation of the renal artery. Hypertension. 2013;61(2):450-6. doi:10.1161 /HYPERTENSIONAHA.111.00095. This study suggests that indetification of the physiological responses to elctrical stimulation of the renal nerves could improve the selection of ablation sites.

18. Sakakura K, Ladich E, Cheng Q, Otsuka F, Yahagi K, Fowler DR, et al. Anatomic assessment of sympathetic peri-arterial renal nerves in man. J Am Coll Cardiol. 2014;64(7):635-43. doi:10.1016/j. jacc.2014.03.059.

19. Roy AK, Fabre A, Cunningham M, Buckley U, Crotty T, Keane D. Post mortem study of the depth and circumferential location of sympathetic nerves in human renal arteries - implications for renal denervation catheter design. Catheter Cardiovasc Interv. 2015;86(2):E32-7. doi:10.1002/ccd.26035.

20. van Amsterdam WA, Blankestijn PJ, Goldschmeding R, Bleys RL. The morphological substrate for renal denervation: nerve distribution patterns and parasympathetic nerves. A post-mortem histological study. Ann Anat. 2016;204:71-9. doi:10.1016/j. aanat.2015.11.004.

21. Mulder J, Hokfelt T, Knuepfer MM, Kopp UC. Renal sensory and sympathetic nerves reinnervate the kidney in a similar timedependent fashion after renal denervation in rats. Am J Physiol Regul Integr Comp Physiol. 2013;304(8):R675-82. doi:10.1152 /ajpregu.00599.2012.

22. Booth LC, Nishi EE, Yao ST, Ramchandra R, Lambert GW, Schlaich MP, et al. Reinnervation of renal afferent and efferent nerves at 5.5 and 11 months after catheter-based radiofrequency renal denervation in sheep. Hypertension. 2015;65(2):393-400. doi:10.1161/HYPERTENSIONAHA.114.04176. This study reports the functional reinnervation of the renal sympathetic nerves following cather-based denervation within a 5.5-month time frame.

23.• Foss JD, Wainford RD, Engeland WC, Fink GD, Osborn JW. A novel method of selective ablation of afferent renal nerves by periaxonal application of capsaicin. Am J Physiol Regul Integr Comp Physiol. 2015;308(2):R112-22. doi:10.1152 /ajpregu.00427.2014. This study provides a novel techique to selective ablate the renal affernet nerves providing for the first time the ability to distinguish the contribution of the renal afferent versus effernet nerves.

24. Foss JD, Fink GD, Osborn JW. Reversal of genetic salt-sensitive hypertension by targeted sympathetic ablation. Hypertension. 2013;61(4):806-11. doi:10.1161/HYPERTENSIONAHA.111.00474.

25. Foss JD, Fink GD, Osborn JW. Differential role of afferent and efferent renal nerves in the maintenance of early- and late-phase Dahl S hypertension. Am J Physiol Regul Integr Comp Physiol. 2016;310(3):R262-7. doi:10.1152/ajpregu.00408.2015.

26. Xiao L, Kirabo A. Wu j, Saleh MA, Zhu L, Wang F et al. Renal denervation prevents immune cell activation and renal inflammation in angiotensin-II induced hypertension. Circ Res. 2015;117(6): 547-57. doi:10.1161/CIRCRESAHA.115.306010.

27. Booth LC, May CN, Yao ST. The role of the renal afferent and efferent nerve fibers in heart failure. Front Physiol. 2015;6:270. doi:10.3389/fphys.2015.00270.

28. Ramchandra R, Hood SG, Frithiof R, McKinley MJ, May CN. The role of the paraventricular nucleus of the hypothalamus in the regulation of cardiac and renal sympathetic nerve activity in conscious normal and heart failure sheep. J Physiol. 2013;591(1):93-107. doi:10.1113/jphysiol.2012.236059.

29. Xu B, Zheng H, Patel KP. Enhanced activation of RVLMprojecting PVN neurons in rats with chronic heart failure. Am J Physiol Heart Circ Physiol. 2012;302(8):H1700-11. doi:10.1152 /ajpheart.00722.2011.

30. Xu B, Zheng H, Liu X, Patel KP. Activation of afferent renal nerves modulates RVLM-projecting PVN neurons. Am J Physiol Heart Circ Physiol. 2015;308(9):H1103-11. doi:10.1152 /ajpheart.00862.2014. This studies provides evidence that activation of the afferet renal nerves is able to influce the activity of central circuitry that modulates sympathetic outflow.

31. Hu J, Li Y, Cheng W, Yang Z, Wang F, Lv P, et al. A comparison of the efficacy of surgical renal denervation and pharmacologic therapies in post-myocardial infarction heart failure. PLoS One. 2014;9(5):e96996. doi:10.1371/journal.pone.0096996.

32. Torp M, Brond L, Nielsen JB, Nielsen S, Christensen S, Jonassen TE. Effects of renal denervation on the NKCC2 cotransporter in the thick ascending limb of the loop of Henle in rats with congestive heart failure. Acta Physiol (Oxf). 2012;204(3):451-9. doi:10.1111 jj.1748-1716.2011.02351.x.

33. Hu J, Yan Y, Zhou Q, Ji M, Niu C, Hou Y, et al. Effects of renal denervation on the development of post-myocardial infarction heart failure and cardiac autonomic nervous system in rats. Int J Cardiol. 2014;172(3):e414-6. doi:10.1016/j.ijcard.2013.12.254. 
34. Guo Z, Zhao Q, Deng H, Tang Y, Wang X, Dai Z, et al. Renal sympathetic denervation attenuates the ventricular substrate and electrophysiological remodeling in dogs with pacing-induced heart failure. Int J Cardiol. 2014;175(1):185-6. doi:10.1016/j. ijcard.2014.04.189.

35. Schiller AM, Haack KK, Pellegrino PR, Curry PL, Zucker IH. Unilateral renal denervation improves autonomic balance in conscious rabbits with chronic heart failure. Am J Physiol Regul Integr Comp Physiol. 2013;305(8):R886-92. doi:10.1152 /ajpregu.00269.2013.

36. Davies JE, Manisty CH, Petraco R, Barron AJ, Unsworth B, Mayet $\mathrm{J}$, et al. First-in-man safety evaluation of renal denervation for chronic systolic heart failure: primary outcome from REACHPilot study. Int J Cardiol. 2013;162(3):189-92. doi:10.1016/j. ijcard.2012.09.019.

37. Mahfoud F, Urban D, Teller D, Linz D, Stawowy P, Hassel JH, et al. Effect of renal denervation on left ventricular mass and function in patients with resistant hypertension: data from a multi-centre cardiovascular magnetic resonance imaging trial. Eur Heart J. 2014;35(33):2224-31b. doi:10.1093/eurheartj/ehu093.

38. Doltra A, Messroghli D, Stawowy P, Hassel JH, Gebker R, Leppanen $\mathrm{O}$, et al. Potential reduction of interstitial myocardial fibrosis with renal denervation. J Am Heart Assoc. 2014;3(6): e001353. doi:10.1161/JAHA.114.001353.

39. Nguyen TP, Sovari AA, Pezhouman A, Iyer S, Cao H, Ko CY, et al. Increased susceptibility of spontaneously hypertensive rats to ventricular tachyarrhythmias in early hypertension. J Physiol. 2016;594(6):1689-707. doi:10.1113/JP271318.

40. Shen MJ, Zipes DP. Role of the autonomic nervous system in modulating cardiac arrhythmias. Circ Res. 2014;114(6):1004-21. doi:10.1161/CIRCRESAHA.113.302549.

41. Huang B, Yu L, Scherlag BJ, Wang S, He B, Yang K, et al. Left renal nerves stimulation facilitates ischemia-induced ventricular arrhythmia by increasing nerve activity of left stellate ganglion. J Cardiovasc Electrophysiol. 2014;25(11):1249-56. doi:10.1111 /jce. 12498

42. Huang B, Yu L, He B, Lu Z, Wang S, He W, et al. Renal sympathetic denervation modulates ventricular electrophysiology and has a protective effect on ischaemia-induced ventricular arrhythmia. Exp Physiol. 2014;99(11):1467-77. doi:10.1113/expphysiol.2014.082057.

43. Hilbert S, Rogge C, Papageorgiou P, Hindricks G, Bollmann A. Successful single-sided renal denervation in drug-resistant hypertension and ventricular tachycardia. Clin Res Cardiol. 2015;104(3): 279-81. doi:10.1007/s00392-014-0790-3.
44. Remo BF, Preminger M, Bradfield J, Mittal S, Boyle N, Gupta A, et al. Safety and efficacy of renal denervation as a novel treatment of ventricular tachycardia storm in patients with cardiomyopathy. Heart Rhythm. 2014;11(4):541-6. doi:10.1016/j. hrthm.2013.12.038.

45. Scholz EP, Raake P, Thomas D, Vogel B, Katus HA, Blessing E. Rescue renal sympathetic denervation in a patient with ventricular electrical storm refractory to endo- and epicardial catheter ablation. Clin Res Cardiol. 2015;104(1):79-84. doi:10.1007/s00392-0140749-4.

46. Hall ME. do Carmo JM, da Silva AA, Juncos LA, Wang Z, Hall JE. Obesity, hypertension, and chronic kidney disease. Int J Nephrol Renovasc Dis. 2014;7:75-88. doi:10.2147/IJNRD.S39739.

47. Lohmeier TE, Iliescu R, Liu B, Henegar JR, Maric-Bilkan C, Irwin ED. Systemic and renal-specific sympathoinhibition in obesity hypertension. Hypertension. 2012;59(2):331-8. doi:10.1161 /HYPERTENSIONAHA.111.185074.

48. Henegar JR, Zhang Y, De Rama R, Hata C, Hall ME, Hall JE. Catheter-based radiorefrequency renal denervation lowers blood pressure in obese hypertensive dogs. Am J Hypertens. 2014;27(10):1285-92. doi:10.1093/ajh/hpu048.

49. Khan SA, Sattar MZ, Abdullah NA, Rathore HA, Abdulla MH, Ahmad A, et al. Obesity depresses baroreflex control of renal sympathetic nerve activity and heart rate in Sprague Dawley rats: role of the renal innervation. Acta Physiol (Oxf). 2015;214(3):390-401. doi:10.1111/apha.12499.

50. Salman IM, Hildreth CM, Ameer OZ, Phillips JK. Differential contribution of afferent and central pathways to the development of baroreflex dysfunction in chronic kidney disease. Hypertension. 2014;63(4): 804-10. doi:10.1161/HYPERTENSIONAHA.113.02110.

51. Khan SA, Sattar MA, Rathore HA, Abdulla MH, Ud Din Ahmad F, Ahmad A, et al. Renal denervation restores the baroreflex control of renal sympathetic nerve activity and heart rate in Wistar-Kyoto rats with cisplatin-induced renal failure. Acta Physiol (Oxf). 2014;210(3):690-700. doi:10.1111/apha.12237.

52. Mauriello A, Rovella V, Anemona L, Servadei F, Giannini E, Bove $\mathrm{P}$ et al. Increased sympathetic renal innervation in hemodialysis patients is the anatomical substrate of sympathetic hyperactivity in end-stage renal disease. J Am Heart Assoc. 2015;4(12). doi:10.1161 /JAHA.115.002426. This study provides the first evidence of an influence on hypertension on the level of renal sympathetic nerve innervation - that is dependent on arteriolar injury. 